\title{
Histoplasmosis - An Unusual Cause of Spastic Paraplegia in an Immunocompetent Host in Bangladesh
}

\author{
CHAYAN KUMAR SINGHA ${ }^{1}$, ESHITA BISWAS ${ }^{2}$, FERDOUS JAHAN ${ }^{1}$, PRODIP KUMAR BISWAS ${ }^{3}$
}

\begin{abstract}
:
A case of disseminated histoplasmosis in a 62-year immunocompetent healthy male presenting as compressive myelopahty without any pulmonary involvement or travel to endemic zone is described. The patient presented with fever, dull ache pain in the back of the lower chest, weakness of both lower limbs with impairment of emptying of bowel and bladder, physical findings include raised temperature, generalized lymphadenopathy, nontender mild hepatomegaly, spastic paraplegia with sensory level up to D8 and local tenderness over D7 vertebra without any gibbus. Diagnosis was confirmed by presence of histoplasmacapsulatum in the lymph node and soft tissue from the spine. The particular interest of this paper is to present a case of disseminated histoplasmosis especially presenting as spastic paraplegia in an immunocompetent host without pulmonary involvement and illustrates the importance of recognizing the possibility of histoplasmosis in Bangladesh where mimickers of histoplasmosis like tuberculosis is extremely common.
\end{abstract}

Keywords: Histoplasmosis, Disseminated histoplasmosis, HistoplasmaCapsulatum, immocompetent host, spastic paraplegia.

\section{Introduction:}

Histoplasmosis is the most common endemic mycosis in human. It has recently emergedas an important opportunistic infection among humanimmunodeficiency virus (HIV) infected persons living in areas where it is endemic. ${ }^{1}$ Histoplasmacapsulatum is a dimorphic fungus that remains in a mycelial form at ambient temperature, and grows as yeast at body temp. It was first described by Samuel darling in 1906 in an adult patient who presumably died of milliary tuberculosis. $^{2}$

Initial infection is spread via respiratory tract through which it enters the reticuloendothelialsystem and resides in macrophages. Most individual with intact cellular immunity are asymptomatic or have mild pulmonary symptoms. Severe disseminated histoplasmosis (DH) develops in patient with primary or secondary immunodeficiency.

Its presentation varies depending on the size of the inoculums, hosts immune status, and presence of underlying host disease. Approximately $10 \%$ cases of histoplasmosis developinto progressive disseminatedHistoplasmosis. ${ }^{3} \mathrm{DH}$ can be fatal if untreated. In Bangladeshi content, reporting

1. Medical Officer, Department of Medicine, BSMMU

2. Medical officer, Bangladesh Shilpokola Academy

3. Resident, Department of Gastroenterology, BSMMU

Correspondence : Dr. Chayan Kumar Singha, FCPS (Medicine), Medical Officer, Department of Medicine. BSMMU, Dhaka. email: chayanbsmmu@.gmail.com. of DH in medical literature is very rareespecially as the cause of spastic paraplegia.The successfullytreated $1^{\text {st }}$ reported case in Bangladesh was in 1982. ${ }^{4}$ We report our experience to increase awarenessof the clinical spectrum of DH where it is similar to other infection particularly Tuberculosis and metastatic malignancy.

\section{Case Report:}

A 62-year old male non diabetic, farmer was admitted with the complaints of low grade fever for $3 \frac{1}{2}$ months, pain in the back of the lower chest for 2 months, weakness of the both lower limbs with impaired emptying of bowel and bladder for 2 months. His past history was unremarkable, and denied any $\mathrm{H} / \mathrm{O}$ extramarital sexual exposures or contact with any sputum positive pulmonary tuberculosis patient. He was not on any drug that might have induced immunosuppressant. On examination revealed mildanemia, temparature $-99^{0} \mathrm{~F}$, generalized non tender discrete lymphadenopathy. Neurological examination revealed spastic paraplegia with sensory level up to D8 and local tenderness over D7 vertebra without any gibbous. Abdominal examination revealed non tender $4 \mathrm{~cm}$ hepatomegaly,vitals were normal and other systemic examination revealed noabnormality. The patient's laboratory investigation results showed hemoglobin $9.2 \mathrm{gm} / \mathrm{dl}$, total WBC- $7500 / \mathrm{mm}^{3} \mathrm{ESR}-$ $84 \mathrm{~mm}$ in $1^{\text {st }}$-hr,chest X-ray was normal. MT- $4 \mathrm{~mm}$, USG showed mild hepato splenomegaly, serum PSA-3.84, x-ray dorsal spine showed reduced joint space between D7 and 
D8. Lymph node biopsy showed multiple PAS positive fungal budding yeast, microbiological investigation of soft tissue from the back showed-intracellular parasite compatible with histoplasmacapsulatum. MRI of dorsal spine showed reduction of joint space between D7 to D8 and paravertebral soft tissue compressing the spinal cord. HIV screening was negative, CD4-845 cells/micro liter, CD8-543 cells/micro liter. He was treated with neurosurgical explorations and stabilization of vertebrae and started on intravenous amphotericin-B followed by oral itraconazole. One month later of his next follow up his condition was improved markedly.

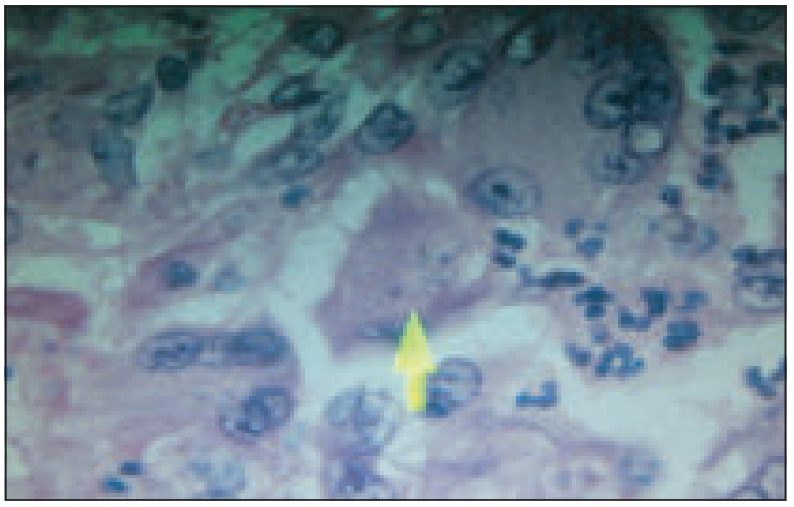

Fig.-1: Showing budding yeast of histoplasma capsulatum.

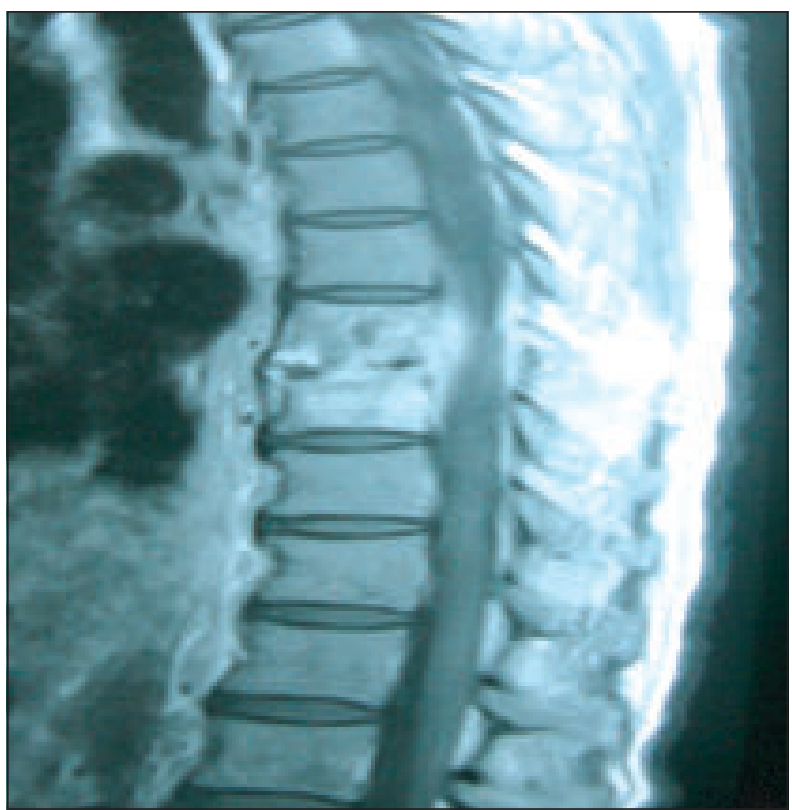

Fig.-2: Showing reduction of disc space with paravertebral soft tissue.

\section{Discussion:}

Histoplasmosis is the most prevalent endemic mycosis in the United States. An estimated 40 million people in the United States have been infected with Histoplasma. capsulatum, with 500,000 new cases occurring in each years. ${ }^{5-7}$ In Bangladesh sporadic cases have been reported in different literature. ${ }^{8-10}$

The causative agenthistoplasmacapsulatum grows in soil laden with excreta of chickens,Pigeons, starlings, blackbirds and bats ${ }^{11}$ Exposure occurs through contact with chicken house, soil containing large amount of bird or bat guano and old houses or caves known to be bat roosts. ${ }^{11}$ Incidence of histoplasmosis in non-endemic areas is far fewer than endemic areas. Commonly, histoplasomosisoccur in non endemic areas due to travel orresidencein endemic areas.

Disseminated histoplasmosis may present either as self limiteddisease orprogressivedisseminated $\mathrm{H}^{12}$ The selflimited condition is found in acute histoplasmosis. Histoplasmosisoccurs during the firstfewweeks of illness when hematogenousdissemination leads to development of various clinical features like hepatomegaly, splenomegaly, bonemarrowsuppression, elevated hepatic enzymes are some of the features. Calcified granuloma in the spleen is a common finding in people living in the endemic zone. Blood culture is rarely positive in acute stage. Specificcell mediatedimmunity plays an important role in controlling the infection in lung and extra pulmonary tissues.

Progressive disseminated histoplasmosis (PDH) usually occurs either in patients at extremes of age or patients with an underlying immune deficiency states. Not all patients harbor an immunodeficient status. ${ }^{12}$ Unidentified mechanism leading to immune deficiency is thoughtto be behind these cases. Ongoing research has identified defects in interferonalpha/interlukin-12 pathway as a possible explanation in otherwise healthy individuals who develop PDH. ${ }^{13}$ Approximately $10 \%$ cases of histoplasmosis develop $\mathrm{PDH} .{ }^{13}$ In $80 \%$ of patients, symptoms are nonspecific and include fever, chills, myalgia, nonproductive cough, and chest pain. The acute syndrome can range from mild (lasting 1-5 days) to severe (lasting 10-21 days); the latter is associated with weight loss, fatigue and night sweats. Fatigue may persist for weeksafter the acute symptoms resolve. Patient may develop a variety of clinical manifestations which include fever, weight loss, fatigue, respiratory complaints like cough and shortnessof breath. Hepatomegaly, splenomegaly, lymphadenopethy, bone marrow involvement are found in less than $50 \%$ of cases. Central nervous system involvement occurs in 5 to 20 percent of cases. ${ }^{13}$ Other less common manifestation include oropharyngeal ulcers, gastrointestinal hemorrhage, adrenal insufficiency, subacute to chronic meningitis and endocarditis, pleuritis, pneumonitis, pancreatitis, cholecystitis, osteomylitis, septic arthritis and epididymitis. Few patients may present with acute shock 
like episodes with hypotension and coagulopathy. Rarely disseminatedhistoplasmosis can cause hypercalcemia due to calcitriol production by activated macrophage, which may lead to the incorrect diagnosis of sarcoidosis.

Histoplasmosis can be diagnosed by growth of histoplasma in culture, fungal stains (Bone marrowaspirate, peripheral blood Smear, lymph node biopsy, bronchoalveolar, lavage fluid, transbronchial biopsy, cutanious biopsy oraccording to the affected site) ${ }^{14}$ Serologic test for antibodies and antigen detection. Skin testing is rarely useful as a diagnostic measure because of high positivity in endemic areas and false-negative results associated with chronic pulmonary and disseminated disease ${ }^{14}$. Among all these, bone marrow examination has the highest diagnostic yeild. ${ }^{14}$ Antigen detection in urine and serum by radioimmunoassay is useful in immunocompromisedpatient when antibody production may be impaired. It is highly sensitive in disseminated infection. It can also be used for monitoring response to treatment especially in AIDS patient the most common finding on chest imaging is diffuse interstitial or reticulonodular infiltrates and milliary infiltrates. Chest Xray in chronic pulmonary histoplasmosis may also show upper lobe involvement with cavitations leading to misdiagnosis of pulmonarytuberculosis ${ }^{14}$.

The recommendedtreatment regimen for disseminated histoplasmosis is liposomal Amphotericin B for 1-2 weeks followed by oral itraconazole for at least 12 months ${ }^{15}$.Suppressive therapy with itraconazole may be required in immunocompromisedpatients like AIDS on organ transplant patient. ${ }^{16}$

Our patient presented with fever, back pain followed by lower limb weakness with impaired bowel and bladder emptying. Before admission his symptoms and imaging findings lead to clinical diagnosis of disseminated tuberculosis involving spine and lymph node which is widely prevalent in Bangladesh. However lymph node biopsy and soft tissue from back of the spine showed histoplasmacapsulatum. Subsequently he was managed accordingly. Indeed histoplasmosis is under reported from Bangladesh due to low index of suspicion and lack of diagnostic facility. Another possible explanation of under diagnosis is that disseminated histoplasmosis resembles visceral leishmaniasis in many aspects of same clinical features. Moreover, both of the two condition responsive to Amphotericin B.

\section{Conclusion:}

This case illustrates the importance of high index of suspicion for diagnosing histoplasmosis in a patient presenting with features suggestive of tuberculosis and sometimes when fail to respond to anti tubercular therapy after a reasonable period of time. Increasing prevalence of AIDS, Organ transplantation and expanding international travel is likely to heighten the possibility of histoplasmosis.n Bangladesh physician need to alert them to this under diagnosed infectious disease which is ultimately fatal if left untreated.

\section{References:}

1. Cano MV, Hajjeh RA. The epidemiology of histoplasmosis: aReview. SeminRespir Infect 2001; 16(2):109-18.

2. Subramanian S, Abraham OC, Rupali P, etal.Disseminated Histoplasmosis. J AssocPhysiciansIndia 2005; 53:185-189.

3. Joshi SA, Kagal AS, Bharadwaj RS, etal. Disseminated Histoplasmosis. Indian J MedMicrobiol 2006; 24:297-298.

4. Islam N, Chowdhury NA. Histoplasmosis from Bangladesh: aCase report. Bangladesh Med Res Counc Bull 1982; 8(1): 21-24.

5. Conces DJ Jr. Histoplasmosis. SeminRoentgenol 1996; 31:14-27.

6. Benenson AS, ed. Control of communicable diseases manual.16th ed. Washington DC: American Public Health Association1995, pp. 237-240.

7. Springston JP. The birds.Occup Health Saf 1998; 67:86-9.

8. Rappo U, Beitler UR, Faulhaber JR, et al. expanding thehorizons of histoplasmosis: disseminated histoplasmosis in arenal transplant patient aftera trip to Bangladesh. TransplInfect Dis 2009; 1-6.

9. Mahbub MS, Ahsan MN, Miah MT, Alam MB, Gupta RD,Arif KM, Hasnain M: Disseminated histoplasmosis. J Medicine2010; 11:70-73.

10. Rahman MM, Hossain SM, Faiz MA, Rahman J, Sultan MT,Dewanjee AK: Disseminated Histoplanmosis in AIDS. J.BCPS2005; 23: 43-45.

11. Rappo U, Beitler UR, Faulhaber JR, et al. expanding the horizons of histoplasmosis: disseminated histoplasmosis in a renal transplant patient after a trip to Bangladesh. Transpl Infect Dis 2009;:1-6.

12. Wheat LJ. Histoplasmosis: a review for clinicians from nonendemic areas. Mycoses 2006; 49:274-282.

13. Wheat LJ, Conger NG. Histoplasmosis.In:HospenthalDR, Rinaldi MG, eds.DiagnosisandTreatment of Human Mycoses. New Jersey: HumanaPress, 2008:318-329.

14. Doughan A. Disseminated histoplasmosis: Case report andbrief review. Travel Med Infect Dis 2006; 4:332-35.

16. Wheat LJ, Freifeld AG, Kleiman MB, et al. Clinical practice guidelines for the management of patients with histoplasmosis: 2007 update by the InfectiousDiseases Society of America. Clin Infect Dis 2007; 45:807-825. 